\title{
Académie suisse pour la qualité en médecine (ASQM)
}

\author{
Roxane Küblera, Esther Kraft ${ }^{\mathrm{b}}$, Christoph Bosshard ${ }^{\mathrm{c}}$ \\ ${ }^{a}$ MA, responsable opérationnelle de I'Académie suisse pour la qualité en médecine (ASQM) de la FMH; 'b lic. rer. oec., cheffe de la division Données,
} démographie et qualité (DDQ) de la FMH; ${ }^{\circ} \mathrm{Dr}$ méd., vice-président de la FMH, responsable du département Données, démographie et qualité

Depuis plus de cinq ans, l'ASQM de la FMH s'engage en faveur de la qualité en médecine par le biais de projets mettant l'accent sur l'intérêt des patients ainsi que sur l'assurance et le développement de la qualité. L'ASQM offre également aux médecins et organisations partenaires différents services dans le domaine de la qualité médicale.

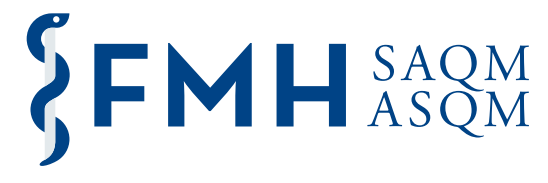

En 2012, la FMH fondait l'Académie suisse pour la qualité en médecine (ASQM). Compétente pour tous les domaines de la qualité en médecine, l'organisation du corps médical suisse s'investit pleinement dans le développement de la qualité de l'activité médicale. Elle

- favorise la promotion de tous les aspects liés à la qualité médicale dans l'intérêt des patients, des proches et des médecins;

- soutient le développement d'une culture de la qualité et s'engage pour un standard élevé lors de la saisie, de l'évaluation et de l'interprétation de données qualité et lors de projets relatifs à la qualité;

- promeut la cohésion du corps médical en matière de qualité et soutient la mise en réseau des activités qualité des diverses sociétés de discipline;

- s'engage pour une meilleure prise en compte de la qualité dans la formation prégraduée, postgraduée et continue des médecins;

- associe les partenaires du domaine de la santé;

- coordonne les aspects médicaux de la recherche concomitante et sur la fourniture des soins et intervient comme interlocutrice dans ce domaine, et négocie les questions de qualité sur le plan national;

- communique sur des thèmes liés à la qualité au sein du corps médical et à l'externe, et représente celuici dans le domaine de la qualité.

L'ASQM veille à associer les délégués à la qualité des organisations représentées à la Chambre médicale à ses travaux (Forum ASQM), et favorise l'échange avec les partenaires de la santé (Dialogue ASQM). Toutes les organisations intéressées et groupes d'intérêts sont invités à siéger au Dialogue Qualité. Toute candidature est la bienvenue (asqm[at]fmh.ch).

\section{Projets qualité de l'ASQM en 2016}

L'ASQM propose de développer la qualité médicale par des projets qui lui sont propres de même que par le soutien et l'accompagnement de projets externes. En 2016, elle a notamment participé aux projets suivants:

(1) Itinéraire clinique des patients atteints du cancer colorectal

Les progrès de la médecine et la spécialisation professionnelle augmentent le nombre d'intervenants spécialisés impliqués dans les diverses étapes d'un traitement. Les patients sont ainsi soit ponctuellement, soit parallèlement, en rapport avec différents spécialistes au cours de leur prise en charge.

Aux côtés de 20 sociétés de discipline médicale et autres groupes professionnels, l'ASQM s'est donné pour mission de développer un itinéraire clinique et multidisciplinaire dans le cadre d'un projet-pilote consacré au cancer colorectal.

(2) Recommandations communes pour les registres de santé

Les registres offrant des données fiables revêtent toujours plus d'importance dans le milieu de la santé et leur nombre ne cesse de croître avec l'augmentation du volume de données. Soucieuses de garantir leur qualité, les organisations $\mathrm{FMH}, \mathrm{ANQ}^{1}$, $\mathrm{H}^{2}{ }^{2} \mathrm{ASSM}^{3}$ et unimedsuisse ${ }^{4}$ ont publié des recommandations communes concernant la création et la gestion de registres dans le domaine de la santé. Ces recommandations contiennent des standards mini- 
maux en matière de protection et de qualité des données, et fournissent également des informations permettant de vérifier la qualité des registres médicaux avant et/ou après leur création (www.asqm.ch $\rightarrow$ Publications $\rightarrow$ Recommandations registres).

(3) Procédure interprofessionnelle de révision par des pairs

Ce concept vise à introduire une procédure uniformisée à l'échelle nationale, qui pourra être utilisée dans les situations statistiquement significatives, et prévoit que les hôpitaux analysent les dossiers des patients avec des pairs externes (confrères) afin d'identifier le potentiel d'amélioration des procédures thérapeutiques. Cette approche interprofessionnelle doit permettre d'améliorer la qualité et la sécurité des patients grâce à des solutions interhospitalières et interdisciplinaires, tout en apportant des améliorations pour toutes les professions impliquées.

(4) Soutien scientifique et financier aux projets de la Fondation Sécurité des patients Suisse

En sa qualité de membre fondateur de la Fondation Sécurité des patients Suisse, la FMH soutient l'organisation aussi bien sur le plan scientifique que financier. En 2015/2016, la Fondation s'est intéressée à la sécurité des patients dans les soins psychiatriques à travers le projet «La sécurité des patients en psychiatrie - état des lieux et plan d'action", qui a visé à présenter les prochaines étapes permettant d'améliorer la sécurité des patients dans les soins psychiatriques dans les années qui viennent. Pour avoir véritablement un impact et sensibiliser l'opinion à cette question, la Fondation a diffusé le plan d'action 2016 (http://www.patientensicherheit.ch/fr/th-mes/Iden tification-des-risques/La-s-curit--des-patients-en-psy chiatrie.html) dans trois langues nationales auprès des principales associations professionnelles, sociétés de discipline médicale et organisations de formation.

(5) Documents de base et prises de position sur la surconsommation de prestations médicales et les campagnes "Choosing Wisely"

En se fondant sur la littérature scientifique, l'ASQM rédige des documents de base sur différents thèmes relatifs à la qualité. Publiés dans le Bulletin des médecins suisses, ils servent de base à la FMH pour se prononcer officiellement dans un document intitulé «Prise de position de la FMH». En 2016, elle a publié des documents de base et prises de position sur «La surconsommation de prestations médicales: un problème de qualité» (http://www.fmh.ch/files/pdf17/ SAEZ_7_GLP_Overuse_F.pdf) et "Choosing Wisely pour diminuer les prestations inutiles (http://www. fmh.ch/files/pdf17/SAEZ_7_GLP_Overuse_F.pdf)».
Vous trouverez des informations détaillées sur ces projets et sur les autres projets de l'ASQM sur notre site Internet: www.asqm.ch $\rightarrow$ Projets relatifs à la qualité.

\section{Charte qualité de l'ASQM}

Elaborée pendant deux ans par les délégués du Forum Qualité de l'ASQM, la charte qualité formalise par écrit les principes politiques de la qualité en médecine et s'adresse aux organisations médicales. En signant cette charte, ces dernières confirment leur volonté commune de renforcer la coopération et la coordination au sein du corps médical ainsi qu'avec d'autres groupes de professionnels de la santé dans les questions relatives à la qualité. La charte repose sur trois piliers: 1) la transparence - les organisations rendent visibles leurs activités en faveur de la qualité; 2) le caractère obligatoireles organisations développent une stratégie en faveur de la qualité et font régulièrement le point dans un rapport qualité; 3) la durabilité - les organisations garantissent le développement de la qualité.

La charte a été adoptée en 2016 après avoir été mise en consultation auprès de toutes les organisations représentées à la Chambre médicale. A ce jour, 67 organisations médicales l'ont d'ores et déjà signée témoignant ainsi leur engagement en faveur de la qualité en médecine. Les organisations signataires vont à présent s'atteler à traduire le contenu de la charte dans une stratégie qualité et un premier rapport dédié à la qualité.

La signature de la charte qualité reste facultative et il est toujours possible de la signer à tout moment. Si vous le souhaitez, n'hésitez pas à nous contacter, le mieux par courriel à asqm[at]fmh.ch.

\section{Services de I'ASOM en faveur}

\section{de la qualité}

Les services de I'ASOM sont à la disposition des organisations médicales et partenaires, des particuliers et des tiers. Voici comment nous pouvons vous soutenir:

- Mise en réseau et coordination autour des questions relatives à la qualité médicale

- Soutien spécialisé pour des questions relatives à la qualité

- Soumission de sujets

- Soutien financier ponctuel

Depuis sa création il y a quatre ans, I'ASOM a déjà reçu et traité plus de 60 demandes de soutien. Vous trouverez de plus amples informations à ce sujet sur le site Internet de I'ASOM, rubrique "Soutien» (www.asqm.ch $\rightarrow$ Services $\rightarrow$ Soutien).

\section{Lettre d'information de I'ASOM}

Cinq à six fois par an, la lettre d'information de I'ASQM vous informe par courriel des projets, travaux et dernières publications en date de I'ASOM. Nous nous réjouissons de recevoir votre inscription à l'adresse asqm[at]fmh.ch. 


\section{Sociétés cantonales de médecine}

Aargauischer Ärzteverband

Association des Médecins du canton de Genève

Ordine dei Medici del Cantone Ticino

Société de médecine du canton d'Unterwald

Société de médecine du canton de Bâle-Campagne

Société de médecine du canton de Fribourg

Société de médecine du canton de Lucerne

Société de médecine du canton de Saint-Gall

Société de médecine du canton de Schwytz

Société de médecine du canton de Soleure

Société de médecine du canton de Thurgovie

Société de médecine du canton de Zurich

Société médicale du Valais

Société neuchâteloise de médecine

\section{Sociétés de discipline médicale}

Société suisse d'allergologie et d'immunologie

Société suisse d'anesthésiologie et de réanimation

Société suisse d'hématologie

Société suisse d'infectiologie

Société suisse d'oncologie médicale

Société suisse d'ophtalmologie

Société suisse d'oto-rhino-laryngologie et de chirurgie cervico-faciale

Société suisse d'urologie

Société suisse de cardiologie

Société suisse de chirurgie

Société suisse de chirurgie cardiaque et vasculaire thoracique

Société suisse de chirurgie de la main

Société suisse de chirurgie plastique, reconstructive et esthétique

Société suisse de chirurgie thoracique

Société suisse de chirurgie vasculaire

Société suisse de dermatologie et vénéréologie

Société suisse de gastroentérologie

Société Suisse de génétique médicale

Société suisse de gynécologie et d'obstétrique

Société suisse de médecine du travail

Société suisse de médecine intensive

Société suisse de médecine interne générale

Société suisse de médecine légale

Société suisse de médecine nucléaire

Société suisse de médecine tropicale et de médecine des voyages

Société suisse de neurochirurgie

Société suisse de neurologie

Société suisse de pathologie

Société suisse de pédiatrie

Société suisse de pharmacologie et de toxicologie clinique

Société suisse de pneumologie

Société suisse de psychiatrie et de psychothérapie d'enfants et d'adolescents

Société suisse de psychiatrie et psychothérapie

Société suisse de radiologie

Société suisse de radio-oncologie

Société suisse de rhumatologie 
Swiss Orthopaedics

Swiss Society for Interventional Pain Management

Organisations faîtières, organisations de base et autres sociétés médicales

ASMAC

Association Suisse des Médecins indépendants travaillant en Cliniques privées et Hôpitaux

Collège de médecine de premier recours

$\mathrm{fmCh}$

FMP

FMPP

Médecins de famille et de l'enfance Suisse

medical women switzerland

Réseau Delta

SFSM

Société suisse de médecine d'urgence et de sauvetage

Société suisse de médecine manuelle

Société suisse des médecins-conseils et médecins d'assurances

Union des sociétés suisses de médecine complémentaire

Sur le site www.asqm.ch $\rightarrow$ Charte qualité, vous trouverez de plus amples informations concernant la charte qualité de l'ASQM mais aussi des modèles de stratégie qualité et de rapport qualité, ainsi qu'une galerie photos de la signature de la charte lors de la Chambre médicale en octobre 2016.

Les 67 organisations suivantes ont déjà signé la charte qualité de l'ASQM (liste au 8 mai 2017):

\section{Perspectives 2017}

Cette année encore, l'ASQM se mettra au service des médecins à travers divers projets et thèmes passionnants en lien avec la qualité en médecine:

- Suivi de la mise en œuvre de la charte qualité: l'ASQM soutient les organisations signataires dans le développement de leur stratégie qualité et la rédaction d'un rapport sur leurs activités en leur proposant notamment un service d'assistance qualité. Si les organisations ont souvent déjà fourni un important travail dans ce domaine, il s'agit à présent de structurer de manière explicite tout ce qui a déjà été fait.

- Projet-pilote "Patient Centered Outcome Registry» (PCOR): ce projet porte sur les attentes des patients dans le contexte de maladies concomitantes et de comorbidités. Les étapes définies pour le diagnostic et le traitement ont-elles permis d'atteindre l'objectif fixé par le patient et le médecin? Cet instrument doit permettre de développer des recommandations de bonnes pratiques fondées sur les expériences au quotidien et venir ainsi compléter les guides de pratique.

- Plateforme des initiatives qualité: la plateforme des activités en faveur de la qualité recommandées par les organisations de la FMH sera mise à jour.

- Avec le travail de mise en réseau mené par l'ASQM, tous ces projets, de même que tous les projets en cours ou achevés, seront repris par la FMH pour s'investir dans le cadre de la modification de l'OAMal et du «renforcement de la qualité et de l'économicité». Le large éventail de projets et d'activités illustre parfaitement le dynamisme avec lequel l'ASQM s'implique dans le secteur de la santé et sa position d'interlocuteur privilégié pour toutes les questions liées à la qualité en médecine. Avec, au centre, la plus-value qu'offre ce travail de qualité pour les patients, les proches et le corps médical.

Pour de plus amples informations: www.asqm.ch

Crédit illustration logo ASQM

Source: FMH 